\title{
Accelerated local dynamics in matrix-free polymer grafted nanoparticles
}

\author{
Mayank Jhalaria, Eileen Buenning, Yucheng Huang, \\ Madhusudan Tyagi, Reiner Zorn, Michaela Zamponi, \\ Victoria García-Sakai, Jacques Jestin, Brian C. Benicewicz \\ and Sanat K. Kumar
}

\section{Published version information}

Citation: M Jhalaria et al. "Accelerated local dynamics in matrix-free polymer grafted nanoparticles." Physical Review Letters, vol. 123, no. 15 (2019): 158003.

DOI: $\underline{10.1103 / P h y s R e v L e t t .123 .158003 ~}$

This version is made available in accordance with publisher policies. Please cite only the published version using the reference above. This is the citation assigned by the publisher at the time of issuing the APV. Please check the publisher's website for any updates. 


\title{
Accelerated Local Dynamics in Matrix-Free Polymer Grafted Nanoparticles
}

\author{
Mayank Jhalaria, ${ }^{1}$ Eileen Buenning, ${ }^{1}$ Yucheng Huang, ${ }^{2}$ Madhusudan Tyagi, ${ }^{3,4}$ Reiner Zorn, ${ }^{5}$ Michaela Zamponi, ${ }^{6}$ \\ Victoria García-Sakai, ${ }^{7}$ Jacques Jestin, ${ }^{8}$ Brian C. Benicewicz, ${ }^{2}$ and Sanat K. Kumar ${ }^{1, *}$ \\ ${ }^{1}$ Department of Chemical Engineering, Columbia University, New York, New York 10027, USA \\ ${ }^{2}$ Department of Chemistry and Biochemistry, University of South Carolina, Columbia, South Carolina 29201, USA \\ ${ }^{3}$ NIST Center for Neutron Research, National Institute of Standards and Technology, Gaithersburg, Maryland 20899-6102, USA \\ ${ }^{4}$ Department of Materials Science and Engineering, University of Maryland, College Park, Maryland 20742, USA \\ ${ }^{5}$ Jülich Centre for Neutron Science (JCNS-1) and Institute for Complex Systems (ICS-1), \\ Forschungszentrum Jülich GmbH, 52425 Jülich, Germany \\ ${ }^{6}$ Jülich Centre for Neutron Science at MLZ, Forschungszentrum Jülich GmbH, Lichtenbergstrasse 1, 85748 Garching, Germany \\ ${ }^{7}$ ISIS Neutron and Muon Source, Rutherford Appleton Laboratory, Chilton, Oxfordshire OX11 OQX, United Kingdom \\ ${ }^{8}$ CEA Saclay, Laboratoire Léon Brillouin, F-91191 Gif Sur Yvette, France
}

(Received 15 May 2019; revised manuscript received 26 July 2019; published 9 October 2019)

\begin{abstract}
The tracer diffusion coefficient of six different permanent gases in polymer-grafted nanoparticle (GNP) membranes, i.e., neat GNP constructs with no solvent, show a maximum as a function of the grafted chain length at fixed grafting density. This trend is reproduced for two different NP sizes and three different polymer chemistries. We postulate that nonmonotonic changes in local, segmental friction as a function of graft chain length (at fixed grafting density) must underpin these effects, and use quasielastic neutron scattering to probe the self-motions of polymer chains at the relevant segmental scale (i.e., sampling local friction or viscosity). These data, when interpreted with a jump diffusion model, show that, in addition to the speeding-up in local chain dynamics, the elementary distance over which segments hop is strongly dependent on graft chain length. We therefore conclude that transport modifications in these GNP layers, which are underpinned by a structural transition from a concentrated brush to semidilute polymer brush, are a consequence of both spatial and temporal changes, both of which are likely driven by the lower polymer densities of the GNPs relative to the neat polymer.
\end{abstract}

DOI: 10.1103/PhysRevLett.123.158003

The addition of nanoparticles (NPs) to a polymer matrix can create materials with dramatically improved thermomechanical properties [1]. However, the tendency of inorganic (hydrophilic) NPs to aggregate in a (hydrophobic) polymer matrix is deleterious to achieving these property improvements. A method to overcome this difficulty is to tether polymer chains onto the NP surface [2] to create polymer-grafted nanoparticles (GNPs). Matrix-free GNPs (no added solvent or free polymer) are one-component systems, and hence have no NP agglomeration issues especially at large grafting densities. They display unique properties such as enhanced gas transport properties [3], ion conduction [4], and hypersonic phononic behavior [5], and are useful in drug delivery [6], self-healing [7], and in the creation of stronger materials $[8,9]$.

While these materials are thus of considerable interest, little is known about how the architecture of an individual GNP (namely the graft density, molecular weight, and core diameter) and the macroscale assemblies they form control multiscale dynamics and properties. Polymers grafted to a spherical surface are particularly unique since the area available to a chain increases as $r^{2}$ ( $r$ is the distance from the NP center)-i.e., the chain feels less crowding from other grafts on the same NP as one moves away from the grafting surface $[10,11]$. This fact underpins a structural transition from a concentrated polymer brush $(\mathrm{CPB}$, where brushes on adjacent NPs do not interpenetrate) to a semidilute polymer brush (SDPB) with increasing graft length at fixed graft density [12].

Previous work from our group has shown that the diffusivity $(D)$ of light gases in pure GNPs displays nonmonotonic behavior as a function of graft molecular weight $\left(M_{n}\right)$ at fixed grafting density $\sigma$, with a maximum in $D$ occurring in the vicinity of the CPB-SDPB transition [3]. For the particular case of $14 \mathrm{~nm}$ diameter spherical silica nanoparticles (NPs) with $\sigma \approx 0.47$ chains $/ \mathrm{nm}^{2}$, this transition occurs for $M_{n} \approx 88 \mathrm{kDa}$. This maximum $D$ can be interpreted as a minimum in local friction experienced by the gas as a function of graft length. Extensive in situ small angle neutron scattering experiments with added solvent show no indication of any spatial heterogeneity in these GNPs [13]. Quasielastic neutron scattering (QENS) experiments on GNPs in dilute solution showed that segmental dynamics in the CPB regions are slower than in the SDPB domain of a grafted polymer layer, i.e., that the CPB has higher local friction [14]. Since these results do not appear 
to explain the $D$ results discussed above, we postulate that studies of neat GNPs (with no added solvent or free polymer) are necessary.

We thus probe the local dynamics of neat GNPs (with no solvent) where the graft $M_{n}$ was varied systematically in a series of experiments. QENS data, which are sensitive to this scale $(\leq 3 \mathrm{~nm})$, when analyzed with a jump diffusion model show that both the frequency and size of jumps in the GNP samples are different from the corresponding polymer melt samples (with no NPs). These results, in combination, explain the remarkable, nonmonotonic transport properties as a function of $M_{n}$, implying that grafting has an effect on both spatial and temporal behavior of the graft polymers.

Spherical $\mathrm{SiO}_{2}$ NPs (14 $\pm 4 \mathrm{~nm}$ diameter, Nissan) were functionalized with poly(methylacrylate) brushes at $\sigma \approx$ 0.47 chains $/ \mathrm{nm}^{2}$ using surface-initiated reversible-addition-fragmentation-chain-transfer polymerization [15]. Further details on the functionalization procedure can be found in the Supplemental Material [16]. A series of samples with differing $M_{n}-29,41,61,80,88,100,136 \mathrm{kDa}$, respectively, were tested (more details in Fig. S3, Table S1). A single pure polymer sample $\left(M_{n} \approx 96 \mathrm{kDa}\right)$, with no added NPs, was deemed sufficient to characterize the ungrafted, unfilled polymer analog; no changes are found in local dynamics as a function of chain length in this range $[19,20]$.

Incoherent neutron scattering was performed on the high flux backscattering spectrometer (HFBS, $q \approx 2-15 \mathrm{~nm}^{-1}$, $t<2000 \mathrm{ps)}$ at the NIST Center of Neutron Research [21], on SPHERES, the spectrometer for high energy resolution $\left(q \approx 3-17 \mathrm{~nm}^{-1}, t<2500 \mathrm{ps}\right)$ operated by the Jülich Centre for Neutron Science (JCNS) at the Heinz MaierLeibnitz Zentrum [22], and on IRIS $\left(q \approx 5-18 \mathrm{~nm}^{-1}\right.$, $t<150 \mathrm{ps})$ at the ISIS Neutron and Muon Source [23]. Background contributions from the sample holder were subtracted and the data were normalized via a vanadium reference to account for detector efficiencies. It was then transformed into the time domain and divided by the instrumental resolution function measured at $4 \mathrm{~K}$ to obtain the intermediate scattering function. All scans were performed at $420 \mathrm{~K}$, which is $\approx 130 \mathrm{~K}$ above the glass transition temperature of the bulk polymer and the composites ( $T_{g} \approx 290 \mathrm{~K}$ for all samples). Sample thicknesses were optimized to ensure that the neutron transmission was always $>90 \%$. The data were reduced using the software DAVE [24], MANTID [25], and SLAW [26], respectively.

Although the coherent scattering length density of silica $\left(\rho_{\mathrm{coh}, \mathrm{SiO}_{2}} \sim 3.4 \times 10^{10} \mathrm{~cm}^{-2}\right)$ is relatively high, the incoherent scattering from the hydrogen atoms along the polymer chains $\left(\rho_{\text {inc,PMA }} \sim 19 \times 10^{10} \mathrm{~cm}^{-2}\right)$ still accounts for greater than $90 \%$ of the total scattering even for GNP samples containing more than $10 \mathrm{vol} \% \mathrm{SiO}_{2}$. Integrated elastic intensities from IRIS confirm that no structural features are observable in the $q$ range of interest, ensuring that the motions observed in the dynamic structure factor are due to polymer self-motion (see Supplemental Material [16], Fig. S2). Figure 1(a) shows a representative spectrum obtained for a GNP with $M_{n} \approx 88 \mathrm{kDa}$ and the reference polymer at a single wave vector, $q=11.1 \mathrm{~nm}^{-1}$ collected on HFBS. (Further comparisons are shown in Fig. S4.) The composite data are broader than the bulk PMA, indicating a larger extent of relaxation within the instrumental time window for the grafted chains. This fact, of a speeding-up of relaxation on grafting, is a major result of this work.

Figure 1(b) shows the transformed spectra for $q$ values varying from $2 \mathrm{~nm}^{-1}$ up to $14.2 \mathrm{~nm}^{-1}$ for $M_{n} \approx 88 \mathrm{kDa}$. The data were fit to the function: $I(q, t)=$ $A(q) \exp \left\{-[t / \tau(q)]^{\beta}\right\}+C$ with a stretching factor $\beta$ while the background term $C$ represents the contribution from
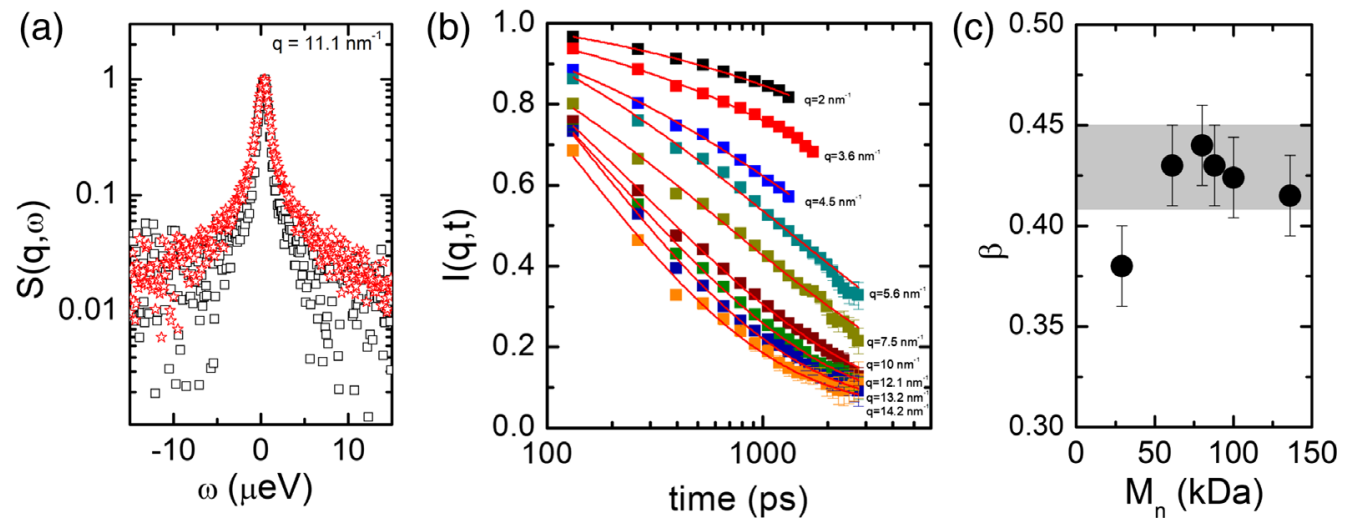

FIG. 1. (a) Dynamic structure factors measured on HFBS for a composite with $M_{n} \approx 88 \mathrm{kDa}$ (red star) and bulk PMA (black square) at $q=11.1 \mathrm{~nm}^{-1}$ showing differences in broadening. The maximum value of the scattering function was normalized to unity in each case to account for differences in the sample thickness and total scattering. (b) Intermediate scattering functions for the $M_{n} \approx 88 \mathrm{kDa}$ composite from $q=2 \mathrm{~nm}^{-1}$ to $14.2 \mathrm{~nm}^{-1}$ at $420 \mathrm{~K}$. Red lines are fits to a stretched exponential function. (c) Stretching exponents $\beta$ for the grafted chains. The gray region defines the range of stretching exponents observed for the bulk polymer with its corresponding error estimate. The stretching exponents are all within error of each other. 
elastic scattering. For the composite we expect there to be an effective elastic contribution from the NPs and a surface layer of the tethered polymers (due to their extremely slow relaxations). To quantify this contribution, plateau values of the intermediate scattering function at long times $\left(t>2000 \mathrm{ps} ; q>10 \mathrm{~nm}^{-1}\right.$; see Supplemental Material [16], Fig. S7) were used. Based on these numbers, the thickness of this immobile layer is estimated to be $\sim 1 \mathrm{~nm}$, similar in magnitude to that reported for more conventional composites [27]. For the neat polymer a constant $\beta=$ $0.43 \pm 0.02$ was used, in line with Plazek and Ngai's estimate [28]. The stretching exponent does not change appreciably for the grafted chains as shown in Fig. 1(c), unlike observations made for NPs dispersed in a polymer matrix [29]. No $q$ dependence of the stretching factor is observed for all materials used (see Supplemental Material [16], Fig. S6).

We find that the $M_{n} \approx 88 \mathrm{kDa}$ GNP has characteristic relaxation times faster than the corresponding reference polymer for all wave vectors [Fig. 2(a)]. As the $\beta$ values are the same for the grafted and pure (no NP) samples [Fig. 1(c)], this trend signifies faster average relaxation processes for the grafted polymer chains. Further, in all cases, there is a "break" in the data in Fig. 2(a) - for smaller $q$ the relaxation times follow a $q^{-2 / \beta}$ scaling, while for larger $q$, we obtain a $q^{-2}$ scaling consistent with local diffusive motion. The crossover between these two limiting behaviors occurs at smaller wave vectors for the grafted samples relative to neat polymers [Figs. 2(b), S6] indicating that locally diffusive dynamics are apparently persistent to larger length scales in the GNPs. These results indicate that a grafted chain experiences fewer local constraints than the corresponding pure polymer. More justification for this statement will be provided below. There is a caveat that the structure factors only decay to about $0.7-0.8$ for the lower $q$ data over the time scales probed. Thus, the relaxation times deduced at these $q$ values should be seen as lower limits to their actual values.

Extending this analysis to composites with different $M_{n}$ [Fig. 2(c)] shows that the average relaxation times (averaged over a $q$ range of $4-14 \mathrm{~nm}^{-1}$ ) of the grafted polymer chains when normalized by the neat polymer are always less than 1; i.e., the segmental dynamics of the grafted chains are significantly faster than the neat polymer. Further, this acceleration is essentially independent of $M_{n}$, except for the lowest grafted chain which is significantly less sped up. These conclusions are very different in character from the experimentally measured gas diffusivity in these media, which show a strong maximum in the vicinity of a $M_{n} \approx 88 \mathrm{kDa}$. In fact, we expect that the gas dynamics should be slave to the relevant friction (or local viscosity) which are controlled by local chain dynamics. The mean relaxation time data derived from the QENS in this fashion thus cannot rationalize the trends for gas diffusion.

We now examine the experimental data more carefully to resolve this discrepacy. The dispersion of the relaxation times with a wave vector, e.g., Fig. 2(a), shows two features. (i) A crossover from a scaling of $q^{-2 / \beta}$ to $q^{-2}$ occurring at an intermediate $q$ value. The scaling of relaxation time with the exponent $-2 / \beta$ is a universal feature that has been observed in several polymers and glass formers, originating from the subdiffusive motion of the polymer segments [30,31]. (ii) A nonmonotonic shift in the crossover $q$ values to lower wave vectors for shorter chains; similarly, the crossover shifts to lower $q$ 's when we go from free PMA chains (with no NP) to the GNP samples at the same $M_{n}$. These results clearly imply that there are changes in spatial correlations on grafting, which are ignored when we only consider changes in relaxation times as discussed above. To include this effect, the relaxation time data are fit to a jump diffusion model proposed by Arbe et al. for polymer melts [32,33]: $\tau(q)=$ $\tau_{0}\left[1+\left(1 / q^{2} l_{0}^{2}\right)\right]^{1 / \beta}$, where $\tau_{0}$ is the time between successive
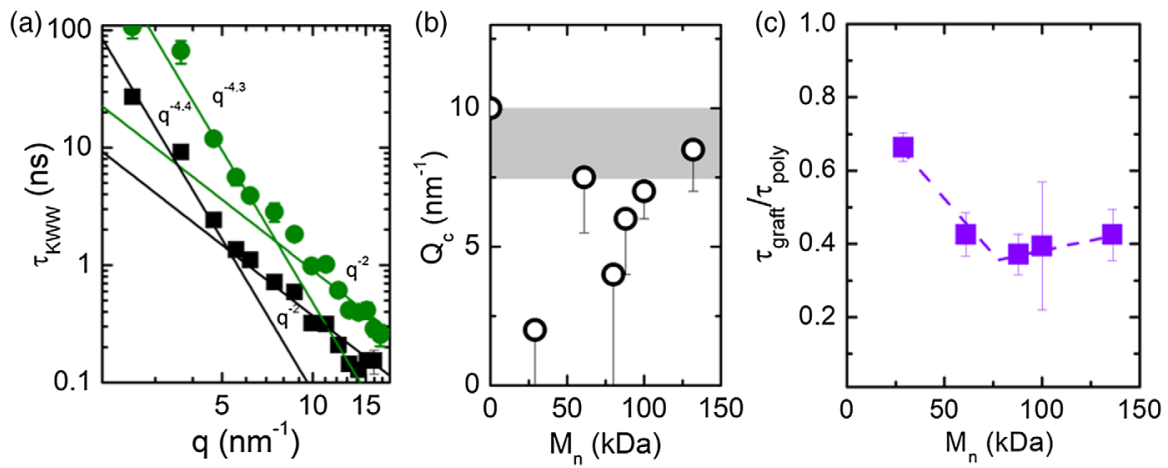

FIG. 2. (a) Comparison of the $q$ dispersion curves for a composite with $M_{n} \approx 88 \mathrm{kDa}$ (black square) and a bulk polymer with $M_{n} \approx 96 \mathrm{kDa}$ (green circle). (b) Crossover $q$ 's (empty circle) for the two asymptotic behaviors observed for the different grafted samples. The gray band corresponds to the crossover wave vectors for the bulk polymer. The $M_{n} \approx 29 \mathrm{kDa}(\mathrm{HFBS})$ and $M_{n} \approx 80 \mathrm{kDa}$ (SPHERES) have no evidence for a crossover within instrument parameters - the error bars extending to zero on the $y$ axis point to this fact. The point at zero molecular weight is the pure PMA (with no NPs). (c) Relaxation times of the composites normalized to the bulk polymer after summing across all wave vectors. The dashed line is a guide to the eye. Data were measured on HFBS. 

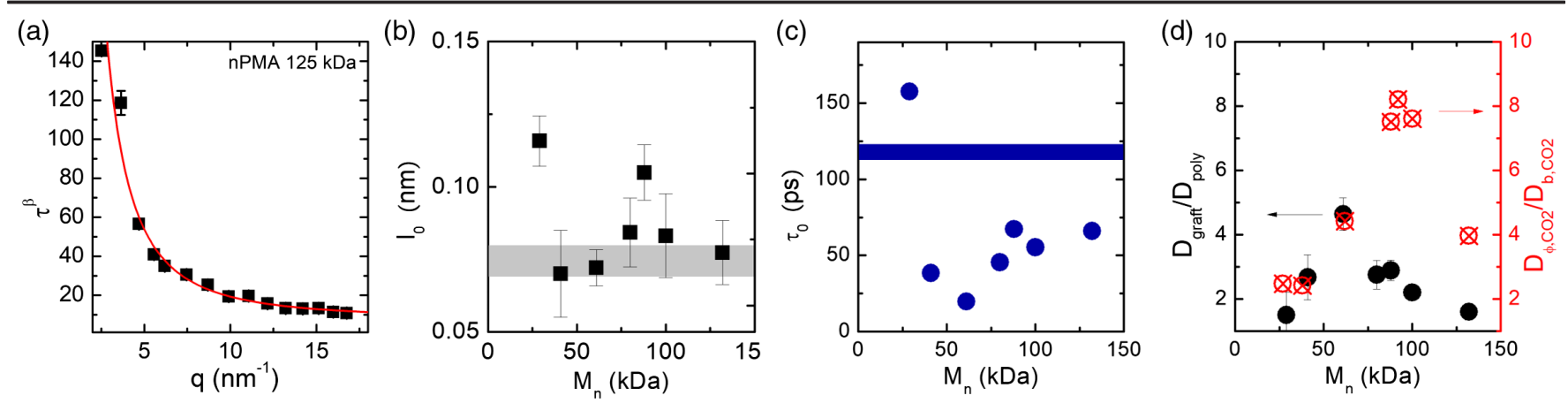

FIG. 3. (a) $\tau^{\beta}$ vs $q$ with fits to the jump diffusion model (solid line). (b) Jump length $l_{0}$ extracted from the model for all composites tested. The gray band corresponds to the jump length for the reference polymer melt (no NPs). (c) Relaxation time $\tau_{0}$ for the diffusive jumps. The bands represent the corresponding fit values for the reference polymer melt. (d) Normalized segmental diffusivity for the composites (black circle). Normalized diffusivities of $\mathrm{CO}_{2}$ in the GNPs are plotted (red symbols) as a function of $M_{n}$. Similar nonmonotonic behavior is observed for the two, although quantitative differences persist.

jumps of characteristic jump length $l_{0}$, which reflects the average distance moved by a participating segment. This model has been successfully used to describe the motions of several polymer melts $[34,35]$ and we can describe our observed relaxation time dispersion with the adjustable parameters $\tau_{0}$ and $l_{0}$ [Figs. 3(a)-3(c)].

Both the jump length and relaxation times are affected by the grafting process - the segments of the grafted polymers execute longer jumps for the $M_{n} \approx 29 \mathrm{kDa}$ and $88 \mathrm{kDa}$ samples than the melt chains with no NPs [Fig. 3(b)]. This jump relaxation could be thought of as a decaging phenomenon, where a relaxational hop opens up a pathway for diffusive transport. Similarly, the relaxation times are much faster $\left(\tau_{0}\right)$ than for the free chains [with no NP, except for the $29 \mathrm{kDa}$ sample, Fig. 3(c)]. The $M_{n} \approx 61 \mathrm{kDa}$ sample, e.g., is 6-7 times faster relative to the neat polymer. While the relaxation time has complicated behavior at low $M_{n}$, it appears to level off beyond the transition to the SDPB regime. A scaled polymeric diffusion constant $D \sim l_{0}^{2} / 6 \tau_{0}$ calculated from these data shows nonmonotonic behavior with a clear maximum at $M_{n} \approx 61 \mathrm{kDa}$ [Fig. 3(d)]. When normalized to the value in the bulk polymer, the effective diffusion constant of the grafted polymer is always greater than 1 and has a peak value $\sim 5$ for the $M_{n} \approx 61 \mathrm{kDa}$ graft; these trends are similar to the nonmonotonic behavior of the diffusion coefficient of $\mathrm{CO}_{2}$ [Fig. 3(d)]. While quantitative differences persist between these segment-level diffusivities and the experimental gas diffusion data, the jump diffusion model data provide a better description of gas transport than a model that only includes changes to relaxation times [Fig. 2(c)]. Parenthetically, we note that there are two possible sources of the quantitative discrepancy between the QENS and the gas diffusion data - the QENS are performed at a higher temperature $(420 \mathrm{~K})$ than the gas transport experiments $(308 \mathrm{~K})$. We know a priori that the response of the diffusion coefficient to temperature changes is not the same for all membranes, thus possibly driving the maximum to another molecular weight (see Supplemental Material, Fig. S1).
Second, there need not be a one-to-one correspondence between the computed local chain diffusion coefficient and the gas diffusion coefficient due to differences in sizes between the two and the connectivity of the segments of the polymer chains.

Based on these results, it is apparent that transport processes must be governed by a combination of larger segmental jumps and faster relaxations. While the mean decaging jump length $l_{0}$ demonstrates less than a twofold variation over the whole range of $M_{n}$ [Fig. 3(b)], it is the distribution of this quantity that shows surprising behavior (see Supplemental Material [16], Fig. S9). We focus particularly on the $M_{n} \approx 88 \mathrm{kDa}$ sample, whose distribution of jump lengths are clearly broader than for all the other grafts (except the $29 \mathrm{kDa}$ sample). This broadening is particularly clear in regions comparable to the sizes of the gas molecules of interest, typical kinetic diameters $>0.3 \mathrm{~nm}$. This finding, which is echoed by PALS measurements [3], implies that it is easier to find microscopic diffusive pathways for gas molecules in the $M_{n} \approx 88 \mathrm{kDa}$ sample. This might help to explain the unusual transport measurements.

We now ask as to the molecular cause of these faster dynamics in the vicinity of the CPB-SDPB transition. For dense short chains grafted on to spherical NPs, the systems have to respect the noninterdigitating nature of brushes on neighboring particles while keeping the system close to spatially uniform in terms of polymer density. We hypothesize that these conflicting requirements lead to an increase in the molar volume of the chains over the reference melt. On increasing the chain length above the CPB-SDPB transition $\left(M_{n} \approx 88 \mathrm{kDa}\right)$, the chains interdigitate because of the increased surface area available to the chains with increasing distance from the NP core. Thus, the total available free volume decreases back towards the neat melt value. This nonmonotonic transport behavior of these GNPs is thus a thermodynamic consequence of the CPBSDPB transition $[12,14,36]$, as has been previously verified through pyconometry measurements [3]. 
Previously, Holt et al. [37], who reported faster segmental relaxations below the glass transition temperature, also emphasized the importance of density changes on grafting chains to NPs. No structural origins of this density decrease were provided. Similarly, Holt and Roland found that the $\beta$ relaxation is accelerated in poly(tetramethyleneoxide-di- $p$-aminobenzoate) oligomers grafted to NPs [38]. These works should be contrasted to the results of Mark et al., who used a combination of neutron spin echo and neutron backscattering on short polyisoprene $\left(M_{n} \approx 5 \mathrm{kDa}\right)$ based GNPs, to show that local chain dynamics remain unchanged relative to the (ungrafted) polymer melt [39]. While this result agrees with the findings of Kim et al. [40], there are no density measurements in these cases (or corresponding transport data) to clearly identify the causes of this different behavior. Nevertheless, Fig. 3(d) implies that the diffusion enhancement in the GNPs disappears when we consider short chains, and hence we believe that the works of Mark et al. [39] and Kim et al. [40] are in a different region of parameter space than our work.

This GNP scaffold thus provides a facile, controllable way of altering segmental dynamics (and hence the dynamics of an entrained penetrant). More detailed studies are required to understand the effects of grafting density, distribution of relaxation times along the backbone of the chain (via isotopic labeling), crossover to Rouse-like relaxation, and how topological confinement affects segmental relaxations for entangled chains. While we have focused our investigation on only one polymer, based on the behavior of diffusion data for other composite systems [3], we expect similar behavior from other polymer chemistries as well.

Financial support for this research was provided by the National Science Foundation under the DMREF Program (No. CBET-1629502). Access to HFBS was provided by the Center for High Resolution Neutron Scattering, a partnership between the National Institute of Standards and Technology and the National Science Foundation under Agreement No. DMR-1508249. We would like to thank Alejandro Krauskopf for experimental assistance. The identification of any commercial product or trade name does not imply endorsement or recommendation by the National Institute of Standards and Technologies.

* To whom correspondence should be addressed. sk2794@columbia.edu

[1] S. K. Kumar, B. C. Benicewicz, R. A. Vaia, and K. I. Winey, Macromolecules 50, 714 (2017).

[2] S. K. Kumar, N. Jouault, B. Benicewicz, and T. Neely, Macromolecules 46, 3199 (2013).

[3] C. R. Bilchak et al., Macromolecules 50, 7111 (2017).

[4] S. Choudhury, S. Stalin, Y. Deng, and L. A. Archer, Chem. Mater. 30, 5996 (2018).
[5] E. Alonso-Redondo, M. Schmitt, Z. Urbach, C. M. Hui, R. Sainidou, P. Rembert, K. Matyjaszewski, M. R. Bockstaller, and G. Fytas, Nat. Commun. 6, 8309 (2015).

[6] K. Yang et al., J. Am. Chem. Soc. 140, 4666 (2018).

[7] B. V. S. Iyer, V. V. Yashin, M. J. Hamer, T. Kowalewski, K. Matyjaszewski, and A. C. Balazs, Prog. Polym. Sci. 40, 121 (2015).

[8] Y. Zheng, Z. M. Abbas, A. Sarkar, Z. Marsh, M. Stefik, and B. C. Benicewicz, Polymer 135, 193 (2018).

[9] J. Choi, C. M. Hui, J. Pietrasik, H. C. Dong, K. Matyjaszewski, and M. R. Bockstaller, Soft Matter 8, 4072 (2012).

[10] P. G. Degennes, Macromolecules 13, 1069 (1980).

[11] M. Daoud and J. P. Cotton, J. Phys. (Paris) 43, 531 (1982).

[12] K. Ohno, T. Morinaga, S. Takeno, Y. Tsujii, and T. Fukuda, Macromolecules 40, 9143 (2007).

[13] E. Buenning, J. Jestin, Y. C. Huang, B. C. Benicewicz, C. J. Durning, and S. K. Kumar, ACS Macro Lett. 7, 1051 (2018).

[14] Y. Wei, Y. F. Xu, A. Faraone, and M. J. A. Hore, ACS Macro Lett. 7, 699 (2018).

[15] C. Z. Li and B. C. Benicewicz, Macromolecules 38, 5929 (2005).

[16] See Supplemental Material at http://link.aps.org/supplemental/ 10.1103/PhysRevLett.123.158003 for additional data, including Refs. [17,18].

[17] A. K. Doolittle, J. Appl. Phys. 23, 236 (1952).

[18] A. K. Doolittle, J. Appl. Phys. 22, 1471 (1951).

[19] V. Arrighi, J. Tanchawanich, and M. T. F. Telling, Macromolecules 46, 216 (2013).

[20] M. Mondello, G. S. Grest, E. B. Webb, and P. Peczak, J. Chem. Phys. 109, 798 (1998).

[21] A. Meyer, R. M. Dimeo, P. M. Gehring, and D. A. Neumann, Rev. Sci. Instrum. 74, 2759 (2003).

[22] J. Wuttke et al., Rev. Sci. Instrum. 83, 075109 (2012).

[23] C. J. Carlile and M. A. Adams, Physica (Amsterdam) 182B, 431 (1992).

[24] R. T. Azuah, L. R. Kneller, Y. M. Qiu, P. L. W. TregennaPiggott, C. M. Brown, J. R. D. Copley, and R. M. Dimeo, J. Res. Natl. Inst. Stand. Technol. 114, 341 (2009).

[25] O. Arnold et al., Nucl. Instrum. Methods Phys. Res., Sect. A 764, 156 (2014).

[26] J. Wuttke, SLAW: Neutron scattering histograms to scattering law converter, http://apps.jcns.fz-juelich.de/slaw..

[27] E. J. Bailey, P. J. Griffin, M. Tyagi, and K. I. Winey, Macromolecules 52, 669 (2019).

[28] D. J. Plazek and K. L. Ngai, Macromolecules 24, 1222 (1991).

[29] S. S. Gong, Q. Chen, J. F. Moll, S. K. Kumar, and R. H. Colby, ACS Macro Lett. 3, 773 (2014).

[30] J. Colmenero, A. Arbe, A. Alegria, M. Monkenbusch, and D. Richter, J. Phys. Condens. Matter 11, A363 (1999).

[31] A. Arbe, J. Colmenero, M. Monkenbusch, and D. Richter, Phys. Rev. Lett. 81, 590 (1998).

[32] A. Arbe, J. Colmenero, F. Alvarez, M. Monkenbusch, D. Richter, B. Farago, and B. Frick, Phys. Rev. E 67, 051802 (2003).

[33] A. Arbe, J. Colmenero, F. Alvarez, M. Monkenbusch, D. Richter, B. Farago, and B. Frick, Phys. Rev. Lett. 89, 245701 (2002). 
[34] A. Narros, A. Arbe, F. Alvarez, J. Colmenero, and D. Richter, J. Chem. Phys. 128, 224905 (2008).

[35] A. C. Genix, A. Arbe, F. Alvarez, J. Colmenero, B. Farago, A. Wischnewski, and D. Richter, Macromolecules 39, 6260 (2006).

[36] C. R. Bilchak, Y. C. Huang, B. C. Benicewicz, C. J. Durning, and S. K. Kumar, ACS Macro Lett. 8, 294 (2019).
[37] A. P. Holt, V. Bocharova, S. W. Cheng, A. M. Kisliuk, G. Ehlers, E. Mamontov, V. N. Novikov, and A. P. Sokolov, Phys. Rev. Mater. 1, 062601(R)(2017).

[38] A. P. Holt and C. M. Roland, Soft Matter 14, 8604 (2018).

[39] C. Mark et al., Phys. Rev. Lett. 119, 047801 (2017).

[40] S. A. Kim, R. Mangal, and L. A. Archer, Macromolecules 48, 6280 (2015). 